\title{
XÂY DỰNG CÔNG THỨC ĐÁNH GIÁ ĐỘ CHÍNH XÁC CỦA MÔ HİNH XU THẾ VÀ THUẬT TOÁN NỘI SUY CÁC DỊ THỬ̛̀nG BOUGUER THEO PHƯONG PHÁP KRIGING TỔNG QUÁT
}

\author{
HÀ MINH HOÀ \\ Viện Khoa học Đo đạc và Bản đồ
}

\section{Tóm tắt}

Việc sử dụng các dị thường Bouguer trên các điểm trọng lực để giải quyết nhiệm vu khoa họ kỹ thuật nội suy xác định các giá trị dị thuờng Bouguer trên các đỉnh của các ô chuẩn trong cơ sở dũ liệu dị thuờng trọng lực quốc gia bao gồm việc xây dựng mô hình xu thế của các dị thuờng này và sủ dụng nó để giải quyết bài toán nội suy. Bài báo khoa học này đã giải quyết hai bài toán: Đánh giá độ chính xác của các dị thương Bouguer được xác định tù mô hình xu thế và Hoàn thiện phuơng pháp nội suy dị thường Bouguer được xác định trên các điểm trọng lục vào các đỉnh của các ô chuẩn trong co sơ dư liệu dị thuoòng trọng lục quốc gia theo phương pháp kriging tổng quát dựa trên thuật toán truy hồi với phép biến đổi xoay $T^{T}$. Việc giải quyết hai bài toán này đã cho phép giải quyết nhiệm vu khoa học - kỹ thuật tuoong đối phưc tạp trong việc triển khai xây dựng co sở dũ liệu dị thường trọng lực quốc gia.

\section{1. Đặt vấn đề}

Các giá trị gia tốc lực trọng trường đo được trên mặt vật lý Trái đất thay đổi phụ thuộc vào các vĩ độ trắc địa $\mathrm{B}$ của các điểm trọng lực. Khi giải quyết bài toán quy chiếu các giá trị dị thường trọng lực lên mặt biên của bài toán biên hỗn hợp của Trắc địa vật lý với việc nhận được các giá trị dị thường không khí tự do, thêm vào đó trong các giá trị dị thường không khí tự do đã loại bỏ sự phụ thuộc vào các vĩ độ trắc địa $\mathrm{B}$ của các điểm trọng lực. Tuy nhiên, các giá trị của dị thường không khí tự do vẫn chịu ảnh hưởng của các gia tốc lực hấp dẫn của các khối lượng vật chất địa hình lồi, lõm xung quanh các điểm trọng lực và của khối lượng vật chất địa hình nằm giữa điểm trọng lực và mặt geoid. Khi loại bỏ các khối lượng vật chất nêu trên và quy chiếu các giá trị dị thường không khí tự do xuống mặt geoid, chúng ta sẽ nhận được các giá trị của dị thường Bouguer. Về mặt lý thuyết, các giá trị dị thường Bouguer thay đổi tương đối đồng đều và được sử dụng để giải quyết bài toán nội suy các giá trị dị thường trọng lực vào các đỉnh của các ô chuẩn trong cơ sở dữ liệu dị thường trọng lực quốc gia.

Trong thực tế việc sử dụng các giá trị dị thường trọng lực Bouguer để giải quyết bài toán nội suy chỉ thực hiện trên đất liền đối với các khu vực có các độ cao địa hình không lớn quá $1500 \mathrm{~m}$. Ở các khu vực rừng núi cao với các độ cao địa hình lớn hơn $1500 \mathrm{~m}$ và trên biển, do ảnh hưởng của các hiệu ứng địa hình - đẳng tĩnh, các giá trị dị thường Bouguer thay đổi rất lớn. Vì lý do này để giải quyết bài toán nội suy dị thường trọng lực, thay cho dị thường Bouguer, người ta sử dụng dị thường địa hình - đẳng tĩnh. Vấn đề này sẽ không được nghiên cứu trong bài báo khoa học này.

Trong trường hợp sử dụng các giá trị dị thường Bouguer để giải quyết bài toán nội suy các giá trị dị thường trọng lực, lưu ý rằng khi tính toán dị thường Bouguer đã sử dụng mật độ vật chất trung bình của lớp vỏ Trái đất $\sigma_{c r}=2,67 \mathrm{~g} / \mathrm{cm}^{3}$ trong khi đó tại các vị trí khác nhau trên lớp vỏ Trái đất, mật độ vật chất thực tế không bằng giá trị này. Do đó các các giá trị dị thường Bouguer bị biến thiên

Ngày nhận bài: 23/5/2018, ngày chuyển phản biện: 25/5/2018, ngày chấp nhận phản biện: 04/6/2018, ngày chấp nhận đăng: 08/6/2018 
và được mô hình hóa bởi mô hình mặt xu thế (trend surface) dưới dạng đa thức bậc q (Goad C. C., C. Ch. Tsherning, M. M. Chin, 1984; Marcin Ligas, Marek Kulczycki, 2014):

$$
\overline{\Delta g_{B}(x, y)}=\beta_{0}+x \cdot \beta_{1}+y \cdot \beta_{2}+x \cdot y \cdot \beta_{3}+x^{2} \cdot \beta_{4}+y^{2} \cdot \beta_{5}+\ldots+x^{q} \cdot \beta_{s-1}+y^{q} \cdot \beta_{s},
$$

ở đây $\mathrm{x}, \mathrm{y}$ là các toạ độ phẳng (đơn vị $\mathrm{km}$ ) của điểm tính; $\beta_{0}, \beta_{1}, \beta_{2}, \ldots, \beta_{s}$ là các hệ số cần tìm của mô hình $\mathrm{xu}$ thế (tổng số các hệ số bằng $\mathrm{k}=\mathrm{s}+1$; giá trị trung bình xác suất $\overline{\Delta g_{B}(x, y)}$ của dị thường Bouguer có đơn vị mGal.

Vấn đề thứ nhất được đặt ra là làm thế nào để đánh giá độ chính xác của các giá trị dị thường Bouguer được xác định theo mô hình (1) ?. Vấn đề này sẽ được giải quyết trong bài báo khoa học này.

Mô hình (1) được sử dụng để giải quyết bài toán nội suy các giá trị dị thường trọng lực vào các đỉnh của các ô chuẩn trong cơ sở dữ liệu dị thường trọng lực quốc gia theo phương pháp kriging tổng quát. Trong nhiều tài liệu về trắc địa vật lý, ví dụ Marcin Ligas, Marek Kulczycki, 2014, việc triển khai phương pháp kriging tổng quát được thực hiện nhờ mô hình bình sai điều kiện kèm các ẩn số. Trong tài liệu (Hà Minh Hòa, 2015) đã đề xuất phương hướng triển khai phương pháp kriging tổng quát theo mô hình bình sai gián tiếp kèm điều kiện nhờ thuật toán truy hồi $\mathrm{T}^{-\mathrm{T}}$, cho phép kiểm tra sự có mặt của các giá trị Bouguer thô. Việc hoàn thiện phương hướng nêu trên là vấn đề thứ hai của bài báo khoa học này.

\section{Giải quyết vấn đề}

Đối với điểm trọng lực Bouguer i $(\mathrm{i}=1,2, . ., \mathrm{n})$ với các tọa độ phẳng $x_{i}, y_{i}$, khi ký hiệu vectơ - cột $\beta=\left(\beta_{0}, \beta_{1}, \beta_{2}, \beta_{3}, \ldots, \beta_{s}\right)^{T} \quad$ là vectơ $\mathrm{k}=\mathrm{s}+1$ các ẩn số cần tìm của đa thức (1) bậc q; vectơ hàng $a_{i}=\left(1, x, y, x . y, x^{2}, y^{2}, \ldots\right)$, từ mô hình (1) chúng ta nhận được phương trình số cải chính:

$$
v_{i}=a_{i} \cdot \beta_{k x \mathrm{~d}}-\overline{\Delta g_{B}\left(x_{i}, y_{i}\right)} \text {. }
$$

Không mất tính chất chung, đối với n điểm trọng lực, khi ký hiệu $L_{n x l}$ là vectơ các số hạng tự do của các phương trình số cải chính (2), chúng ta có dạng của hệ phương trình số cải chính:

$$
V_{n x 1}=A_{n x k} \cdot \beta_{k o d}-L_{n x 1} \text {. }
$$

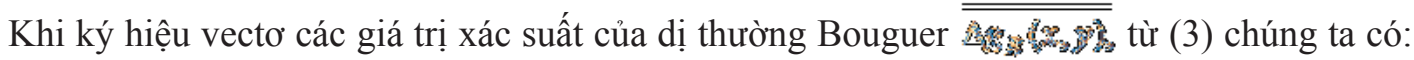

$$
\overline{\overline{\Delta g_{B}(x, y)}}=L+V=A . \beta .
$$

Từ kết quả giải hệ phương trình (3) dưới điều kiện $V^{T} V=$ min chúng ta nhận được vectơ nghiệm:

$$
\beta=\left(A^{T} A\right)^{-1} \cdot A^{T} \cdot L \text {. }
$$

Thay vectơ nghiệm $\beta$ vào (4), chúng ta biểu diễn vectơ các giá trị xác suất của dị thường Bouguer ở dạng sau:

$$
\overline{\overline{\Delta g_{B}(x, y)}}=A \cdot\left(A^{T} A\right)^{-1} \cdot A^{T} \cdot L .
$$

Khi nhận ma trận hiệp phương sai của vectơ bằng ở đây - ma trận đơn vị bậc $\mathrm{n}$, - sai số trung phương đơn vị trọng số, từ (5) suy ra công thức xác định ma trận hiệp phương sai của vectơ các giá trị xác suất nhất của dị thường Bouguer ở dạng sau:

$$
K_{\overline{\Delta g_{B}}}=\sigma^{2} \cdot A \cdot\left(A^{T} A\right)^{-1} \cdot A^{T} \cdot A \cdot\left(A^{T} A\right)^{-1} \cdot A^{T}=\sigma^{2} \cdot A \cdot\left(A^{T} A\right)^{-1} \cdot A^{T} .
$$

Chúng ta ký hiệu $\left(K_{\overline{\Delta g_{B}}}\right)_{i i} . i=1,2, \ldots, n$, là thành phần đường chéo thứ i của ma trận tương quan 
$F_{\overline{M_{g_{B}}}}$ Dựa trên tính chất của vết của ma trận, từ (6) chúng ta có:

$$
\begin{aligned}
& \text { Trace }\left(K_{\overline{\Delta g_{B}}}\right)=\sum_{i=1}^{n}\left(K_{\overline{\Delta g_{B}}}\right)_{i i}=\sigma^{2} \text {.Trace }\left(A \cdot\left(A^{T} A\right)^{-1} \cdot A^{T}\right)= \\
& =\sigma^{2} \text {.Trace }\left(A^{T} A \cdot\left(A^{T} A\right)^{-1}\right)=\sigma^{2} \text {.Trace }\left(E_{k x k}\right)=\sigma^{2} \cdot k .
\end{aligned}
$$

Do ma trận $S=A \cdot\left(A^{T} A\right)^{-1} \cdot A^{T}$ là ma trận lũy đẳng thỏa mãn tính chất $S \cdot S=S$, nên hạng của ma trận $\mathrm{S}$ là $\operatorname{rank}(\mathrm{S})=\mathrm{k}$ bằng vết Trace $(\mathrm{S})=\mathrm{k}$.

Chúng ta coi các giá trị xác suất của dị thường Bouguer $\overline{\overline{\Delta g_{B}(x, y)}}$ có cùng độ chính xác, tức $\left(K \overline{\overline{\Delta g_{B}}}\right)_{11}=\left(K \overline{\overline{\Delta g_{B}}}\right)_{22}=\ldots=\left(K \overline{\overline{\Delta g_{B}}}\right)_{n n}$. Khi đó lưu ý (7) suy ra

$$
\left(K \overline{\overline{\Delta g_{B}}}\right)_{11}=\left(K_{\overline{\Delta g_{B}}}\right)_{22}=\ldots=\left(K \overline{\overline{\Delta g_{B}}}\right)_{n n}=\sigma^{2} \cdot \frac{k}{n}=m \frac{2}{\overline{\Delta g_{B}}} \text {. }
$$

Từ đây chúng ta đánh giá độ chính xác của các giá trị trung bình xác suất từ mô hình xu thế theo công thức sau:

$$
m \overline{\overline{\Delta g_{B}}}= \pm \frac{\sigma}{\sqrt{n / k}}
$$

ở đây sai số trung phương đơn vị trọng số được đánh giá theo công thức: $\quad \sigma= \pm \sqrt{\frac{V^{T} V}{n-k}}$.

Không khó khăn để nhận thấy rằng khi áp dụng lý thuyết nêu ở trên để đánh giá giá trị trung bình xác xuất $A=$ gen của một đại lượng được đo $\mathrm{n}$ lần, với tổng số ẩn số $\mathrm{k}=1$, lưu ý ma trận $A=(1$ $1 \ldots 1)^{T}, A^{T} A=n$, từ các công thức $(5),(8),(9)$ chúng ta sẽ suy ra các công thức đã biết:

sai số trung phương đơn vị trọng số: $\sigma= \pm \sqrt{\frac{\sum_{i=1}^{n}\left(L_{i}-\mu\right)^{2}}{n-1}}$,

sai số trung phương của giá trị trung bình xác suất: $m_{\mu}= \pm \frac{\sigma}{\sqrt{n}}$.

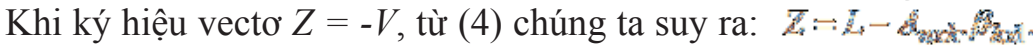

Trong Địa thống kê, vectơ Z (10) được gọi lại vectơ của trường ngẫu nhiên còn dư (residual random field) và được sử dụng rộng rãi trong các phương pháp nội suy collocation, kriging. Bây giờ để tiện trình bày, chúng ta ký hiệu $\mu(x, y)=\overline{\overline{\Delta g_{B}(x, y)}}$ là giá trị xác suất của dị thường Bouguer tại điểm trọng lực $(\mathrm{x}, \mathrm{y})$ được xác định từ mô hình $(4)$, $\mathrm{Q}$ là tập hợp các giá trị dị thường Bouguer trên các điểm trọng lực, $\mathrm{P}$ là tập hợp các điểm nội suy (các đỉnh của các ô chuẩn dị thường trọng lực), $C_{Q}$ là ma trận hiệp phương sai của các giá trị dị thường Bouguer trong tập hợp $\mathrm{Q}$. Đối với điểm nội

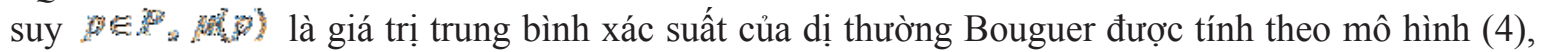
$C_{Q p}$ là ma trận tương quan chéo giữa các dị thường Bouguer trong tập hợp $\mathrm{Q}$ và dị thường Bouguer tại điểm $\mathrm{p}$.

Trong trường hợp chung đối với các phương pháp nội suy collocation, kriging, giá trị nội suy của dị thường Bouguer tại điểm $\mathrm{y}_{3} \mathrm{E}$ được xác định theo công thức: $\widetilde{\mu}(p)=\mu(p)+\lambda^{T} . Z$, ở đây vectơ $\mathrm{Z}$ được xác định theo công thức (10).

Vấn đề được đặt ra là xác định vectơ $\lambda^{T}$ theo công thức (11) đối với mỗi điểm nội suy Khác với cách tiếp cận trong tài liệu (Hà Minh Hòa, 2015), trong bài báo này chúng ta chỉ cần tính đến hai điều kiện đối với vectơ $\lambda^{T}$ như sau: $\lambda^{T} \cdot e=1$

$$
\sum_{i=1}^{n} \mu\left(x_{i}, y_{i}\right) \cdot \lambda_{i}=\mu(P) \text {. }
$$


ở đây vectơ $e_{n x l}=\left(\begin{array}{llll}1 & 1 \ldots 1\end{array}\right)^{T}$.

Khi tìm cực tiểu của hàm $\Phi(\lambda, K)=\lambda^{T} \cdot C_{Q} \lambda+C(0)-2 \cdot \lambda^{T} \cdot C_{Q p}$, ở đây $\mathrm{C}(0)$ là hàm phương sai của vectơ Z, dưới các điều kiện (12), (13), chúng ta nhận được hệ phương trình chuẩn:

$$
\left[\begin{array}{ccccc}
C_{11} & \ldots & C_{1 n} & 1 & \mu\left(x_{1}, y_{1}\right) \\
\cdot & \ldots \ldots \ldots \ldots . . & . & \\
C_{n 1} & \ldots & C_{n n} & 1 & \mu\left(x_{n}, y_{n}\right) \\
1 & \ldots \ldots \ldots .1 & 0 & 0 \\
\mu\left(x_{1}, y_{1}\right) \ldots \mu\left(x_{n}, y_{n}\right) & 0 & 0
\end{array}\right] \cdot\left[\begin{array}{l}
\lambda_{1} \\
\cdot \\
\lambda_{n} \\
K_{1} \\
K_{2}
\end{array}\right]=\left[\begin{array}{l}
C_{1 P} \\
\\
C_{n P} \\
1 \\
\mu(p)
\end{array}\right],
$$

ở đây $K_{1}, K_{2}$ - các nhân tử Lagrange.

Ma trận chuẩn trong hệ (14) là ma trận đối xứng xác định không dương. Điều này gây khó khăn cho việc giải hệ phương trình chuẩn này. Để khắc phục hạn chế này, chúng ta làm như sau. Ký hiệu

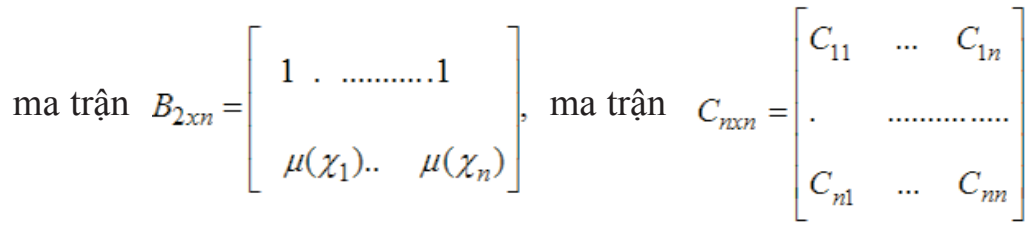

các vectơ con số hạng tự do $C_{Q, p}=\left[\begin{array}{l}C_{1 P} \\ \cdot \\ \cdot \\ C_{n P}\end{array}\right] ; \quad W_{p}=\left[\begin{array}{l}1 \\ \mu\left(\chi_{P}\right)\end{array}\right]$,

hệ phương trình chuẩn (14) có dạng: $\quad\left[\begin{array}{ll}C & B^{T} \\ B & 0\end{array}\right] \cdot\left[\begin{array}{l}\lambda \\ K\end{array}\right]=\left[\begin{array}{l}C_{Q p} \\ W_{p}\end{array}\right]$.

Theo phương pháp viền (Bordering method), khi cho ma trận chuẩn $\bar{R}=\left[\begin{array}{ll}R_{11} & R_{12} \\ R_{21} & R_{22}\end{array}\right]$, ma trận nghịch đảo $\bar{Q}:-\overline{\underline{m}}^{-1}$ của nó có dạng $\bar{Q}=\left[\begin{array}{ll}Q_{11} & Q_{12} \\ Q_{21} & Q_{22}\end{array}\right]$,

ở đây $Q_{11}=R_{11}^{-1}-Q_{12} \cdot R_{21} \cdot R_{11}^{-1} ; Q_{12}=-R_{11}^{-1} \cdot R_{12} \cdot Q_{22} ; Q_{21}=Q_{12}^{T} ; Q_{22}=\left(R_{22}-R_{21} \cdot R_{11}^{-1} \cdot R_{12}\right)^{-1}$.

Đối với ma trận chuẩn $\overline{\mathbb{R}}$ của hệ phương trình chuẩn (17), khi ký hiệu

$$
R_{11}=C, R_{12}=B^{T}=R_{21}^{T}, R_{22}=0 .
$$

từ (18) chúng ta có các thành phần của ma trận nghịch đảo như sau:

$$
\begin{aligned}
Q_{11}=C^{-1}-C^{-1} \cdot B^{T} \cdot\left[B \cdot C^{-1} \cdot B^{T}\right]^{-1} \cdot B \cdot C^{-1} ; Q_{12}=C^{-1} \cdot B^{T} \cdot\left[B \cdot C^{-1} \cdot B^{T}\right]^{-1} ; Q_{21} & =\left[B \cdot C^{-1} \cdot B^{T}\right]^{-1} \cdot B \cdot C^{-1} ; \\
Q_{22} & =-\left[B \cdot C^{-1} \cdot B^{T}\right]^{-1} .
\end{aligned}
$$


Các nghiệm của hệ phương trình chuẩn (17) được xác định từ biểu thức :

$$
\left[\begin{array}{l}
\lambda \\
K
\end{array}\right]=\left[\begin{array}{ll}
Q_{11} & Q_{12} \\
Q_{21} & Q_{22}
\end{array}\right] \cdot\left[\begin{array}{l}
C_{Q p} \\
W_{p}
\end{array}\right] .
$$

Từ (19) chúng ta thấy rằng đối với các biến ngẫu nhiên trên $n$ điểm thuộc tập hợp $\mathrm{Q}$, các ma trận $C$ và $B$ hoàn toàn được xác định và không đổi, tức các thành phần của ma trận nghịch đảo $\bar{x}=\overline{x^{2}}=-1$ là không đổi khi giải quyết bài toán nội suy xác định các dị thường Bouguer trên các điểm thuộc tập hợp $P$ theo phương pháp kriging tổng quát. Do đó để nội suy xác định các dị thường Bouguer tin cậy nhất trên mỗi điểm $p \in P$, chúng ta chỉ việc xác định các vectơ $C_{Q P}$ và $W_{P}$ ở dạng (17) đối với điểm $p \in P$, và xác định vectơ $\lambda$ cho điểm này. Lưu ý (19) từ hệ phương trình trên chúng ta có biểu thức xác định vectơ $\lambda$ ở dạng sau:

$$
\begin{aligned}
& \lambda=Q_{11} \cdot C_{Q p}+Q_{12} \cdot W_{p}=\left\{C^{-1}-C^{-1} \cdot B^{T} \cdot\left[B \cdot C^{-1} \cdot B^{T}\right]^{-1} \cdot B \cdot C^{-1}\right\} \cdot C_{Q p}+C^{-1} \cdot B^{T} \cdot\left[B \cdot C^{-1} \cdot B^{T}\right]^{-1} \cdot W_{p}=\lambda_{1}+\lambda_{2}, \\
& \text { ở đây } \lambda_{1}=\left\{C^{-1}-C^{-1} \cdot B^{T} \cdot\left[B \cdot C^{-1} \cdot B^{T}\right]^{-1} \cdot B \cdot C^{-1}\right\} \cdot C_{Q p} ; \quad(21) \quad \lambda_{2}=C^{-1} \cdot B^{T} \cdot\left[B \cdot C^{-1} \cdot B^{T}\right]^{-1} \cdot W_{p}
\end{aligned}
$$

Để xác định vectơ $\lambda(20)$ với mục đích nội suy xác định biến ngẫu nhiên tin cậy nhất trên mỗi điểm $\lambda_{1}(21)$ và $\lambda_{2}$ (22) ở dưới đây. Từ công thức nghịch đảo ma trận ở dạng (Duncan W.J., 1944):

$$
\left(C+B^{T} \cdot \hat{P} \cdot B\right)^{-1}=C^{-1}-C^{-1} \cdot B^{T} \cdot\left[\hat{P}^{-1}+B \cdot C^{-1} \cdot B^{T}\right]^{-1} \cdot B \cdot C^{-1},
$$

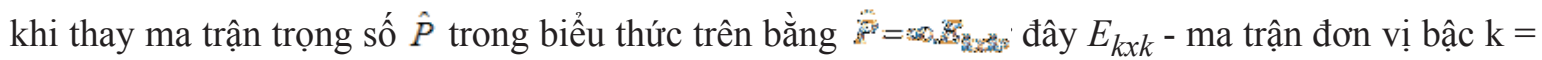
$\mathrm{q}+1$, thì chúng ta có công thức: $\left(C+B^{T} \cdot \hat{P} \cdot B\right)^{-1}=C^{-1}-C^{-1} \cdot B^{T} \cdot\left[B \cdot C^{-1} \cdot B^{T}\right]^{-1} \cdot B \cdot C^{-1}$.

Chúng ta không khó khăn để nhận thấy rằng vectơ $\lambda_{1}(21)$ là vectơ nghiệm của hệ phương trình chuẩn:

$$
\left(C+B^{T} \cdot \hat{P} \cdot B\right) \lambda_{1}=C_{Q p},
$$

thêm vào đó vectơ $\lambda_{1}(21)$ được xác định từ hệ $\lambda_{1}=\left(C+B^{T} \cdot \hat{P} \cdot B\right)^{-1} \cdot C_{Q p}$

Về phần mình, hệ phương trình chuẩn $(23)$ được lập từ hệ phương trình số cải chính:

$$
V_{1}=E_{n x n}, \lambda_{1}-C^{-1} \cdot C_{Q, p}, \quad P_{1}=C, V_{F}=B \cdot \lambda_{1}+0.0, \quad \hat{P}=\infty . E_{k x k}
$$

theo nguyên tắc bình phương nhỏ nhất, ở đây $E_{n x n}$ và $E_{k x k}$ - các ma trận đơn vị bậc $\mathrm{n}$ và bậc $\mathrm{k}=\mathrm{q}+1$ một cách tương ứng.

Hệ phương trình số cải chính (25) là mô hình của bài toán bình sai gián tiếp kèm các điều kiện. Khi biểu diễn ma trận $C^{-1}$ dưới dạng khai triển tam giác $C^{-1}=U^{T}$. $U$, ở đây $\mathrm{U}$ là ma trận tam giác trên bậc $\mathrm{n} x \mathrm{n}$, ma trận $\mathrm{C}$ có dạng: $C=U^{-1} \cdot U^{T}$

Trong trường hợp này, hệ phương trình $(25)$ được biểu diễn lại dưới dạng:

$$
\overline{V_{1}}=U^{-T} \cdot \lambda_{1}-U \cdot C_{Q, p}, \quad \bar{P}_{1}=E_{n x n}, \quad V_{F}=B \cdot \lambda_{1}+0.0, \quad \hat{P}=\infty \cdot E_{k x k}
$$

ở đây vectơ số cải chính $\bar{F}_{1}=\mathbb{R}^{-\pi} F_{W_{n}} E_{n x n}$ là ma trận đơn vị bậc $\mathrm{n} \times \mathrm{n}$.

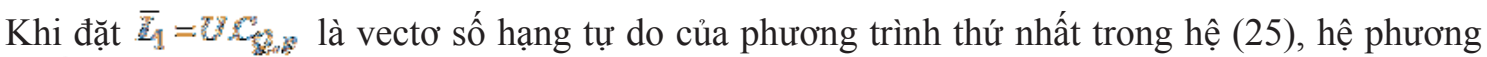
trình số cải chính (25) có dạng mới:

$$
\overline{V_{1}}=U^{-T} \cdot \lambda_{1}-\bar{L}_{1}, \quad \bar{P}_{1}=E_{n x n}, V_{F}=B \cdot \lambda_{1}+0.0, \quad \hat{P}=\infty \cdot E_{k x k}
$$

Hệ phương trình số cải chính (28) dễ dàng được thành lập, khi chúng ta thực hiện biến đổi ma 
trận hiệp phương sai $\mathrm{C}$ thành ma trận tam giác trên $U^{-1}$. Vấn đề này sẽ được nghiên cứu tiếp theo ở phần dưới. Trong trường hợp này, vectơ số hạng tự do $\overline{\bar{L}_{2}}$ được xác định từ hệ phương trình

$$
U^{-1} \cdot \bar{L}_{1}=C_{Q p} \text {. }
$$

Thực chất hệ phương trình số cải chính (28) là mô hình toán học của bài toán bình sai gián tiếp kèm điều kiện, có ma trận chuẩn đối xứng không xác định dương và dễ dàng được giải nhờ thuật toán truy hồi với phép biến đổi xoay $T^{-T}$. Khi giải hệ phương trình số cải chính (28) theo thuật toán truy hồi với phép biến đổi xoay $T^{-T}$, chúng ta nhận được ma trận tam giác dưới $T^{-T}$ thêm vào đó ma trận $T^{-T}$ liên hệ với ma trận nghịch đảo $\left(C+B^{T} \cdot \hat{P} \cdot B\right)^{-1}$ trong công thức (24) dưới dạng:

$$
\left(C+B^{T} \cdot \hat{P} \cdot B\right)^{-1}=T^{-1} \cdot T^{-T}
$$

Khi đặt (30) vào (24) và ký hiệu $\theta_{n \times 1}=T^{-T} \cdot C_{Q p}$,

chúng ta thấy rằng vectơ thành phần $\lambda_{1}$ được xác định từ phương trình: $\lambda_{1}-\omega^{-1}$

Lưu ý các công thức (31), (32) để tính toán vectơ thành phần $\lambda_{1}$ đối với mỗi điểm nội suy chúng ta nhận thấy rằng không cần thiết giải hệ phương trình số cải chính (28). Khi sử dụng thuật toán bình sai truy hồi với phép biến đổi xoay $T^{-T}$, chúng ta tạo ma trận ban đầu $T_{0}^{-T}=U^{-T}$ và đưa phương trình thứ hai trong hệ phương trình (28) vào tính toán truy hồi theo thuật toán nêu trên. Kết quả chúng ta sẽ nhận được ma trận tam giác dưới $T^{-T}$. Tiếp theo, đối với mỗi điểm nội suy chúng ta tính toán vectơ thành phần $\lambda_{1}$ theo các công thức (31), (32).

Đối với điểm $\mathrm{p}$ đầu tiên thuộc tập hợp $P$ chúng ta lập hệ phương trình số cải chính (28) với vectơ số hạng tự do $\bar{L}_{1}$ được xác định từ hệ phương trình (29). Sau khi giải hệ phương trình trên theo thuật toán $T^{-T}$, chúng ta sẽ nhận được ma trận tam giác dưới $T^{-T}$ và vectơ thành phần $\lambda_{1}$ đối với điểm $\mathrm{p}$ đầu tiên thuộc tập hợp $P$. Đối với các điểm $\mathrm{p}$ thứ hai trở đi cho đến điểm thứ $\mathrm{m}$ thuộc tập hợp $P e$, ở $^{\circ}$ đây $\mathrm{m}$ là tổng số điểm thuộc tập hợp $P$, chúng ta chỉ việc xác định các vectơ thành phần $\lambda_{1}$ dựa trên các công thức (31) và (32).

Chúng ta sẽ nghiên cứu tiếp theo phương pháp xác định các các vectơ thành phần $\lambda_{2}$ đối với các

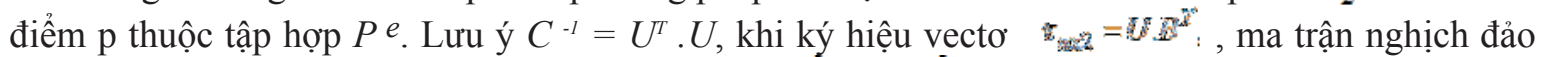
$\left[B \cdot C^{-1} \cdot B^{T}\right]^{-1}$ bậc 2 x 2 được xác định theo công thức: $\left[B \cdot C^{-1} \cdot B^{T}\right]^{-1}=\left(\tau^{T} \tau\right)^{-1}$.

Khi đã xác định được ma trận $U^{-1}$, vectơ $\tau$ được xác định từ hệ phương trình: $Z^{-\pi}{ }^{-\pi}=Z^{T}$.

Đối với mỗi điểm $\mathrm{p}$ thuộc tập hợp $P e$, lưu ý (33) chúng ta thấy rằng vectơ thành phần $\lambda_{2}(22)$ được từ hệ phương trình: $U^{-T} \cdot \lambda_{2}=\tau \cdot\left[\tau^{T} \tau\right]^{-1} W_{p}$,

ở đây vectơ được xác định từ hệ phương trình (34).

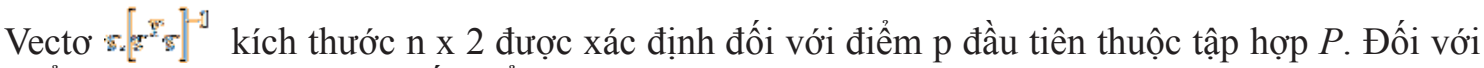
các điểm $\mathrm{p}$ thứ hai trở đi cho đến điểm thứ $\mathrm{m}$ thuộc tập hợp $P$, chúng ta chỉ việc xác định các vectơ thành phần $\lambda_{2}$ dựa trên việc giải hệ phương trình (35), thêm vào đó các vectơ số hạng tự do $W_{p}$ của các điểm này được xác định từ (16).

Vấn đề cuối cùng cần giải quyết là khai triển tam giác ma trận $\mathrm{C}$ (15) bậc $\mathrm{n} \times \mathrm{n}$ dưới dạng (26). Do ma trận $\mathrm{C}$ là ma trận đối xứng, nên chúng ta chỉ thực hiện tính toán với phần tam giác trên của nó. Chúng ta ký hiệu $C_{i, j}$ và $U_{i, j}^{-1}$ là các phần tử nằm ở hàng $\mathrm{i}$, cột $\mathrm{j}$ của ma trận $\mathrm{C}$ và ma trận tam giác trên $U^{-1}$ một cách tương ứng. Việc khai triển được thực hiện theo quy trình sau:

$$
* \mathrm{j}=\mathrm{n}: U_{n, n}^{-1}=\sqrt{C_{n, n}}
$$


Đối với các hàng $\mathrm{i}=1,2, . ., \mathrm{n}-1: \quad U_{i, n}^{-1}=\frac{C_{i, n}}{U_{n, n}^{-1}}$.

* Các cột giảm dần: $\mathrm{j}=\mathrm{n}-1, \mathrm{n}-2, \ldots, 1$

Đối với mỗi cột $\mathrm{j}$, các hàng $\mathrm{i}$ giảm dần từ $\mathrm{j}$ đến 1 thì :

Khi i $=\mathrm{j}: \quad U_{i, i}^{-1}=\sqrt{C_{i, i}-\sum_{j 1=j+1}^{n}\left(U_{i, j 1}^{-1}\right)^{2}}$

Khi j > i: $\quad U_{i, j}^{-1}=\frac{C_{i, j}-\sum_{j 1=j+1}^{n} U_{i, j 1}^{-1} \cdot U_{j, j 1}^{-1}}{U_{j, j}^{-1}}$.

Đến đây chúng ta đã nghiên cứu cơ sở khoa học và quy trình triển khai phương pháp kriging tổng quát để xác định các vectơ phục vụ việc giải quyết bài toán nội suy xác định các giá trị tin cậy nhất của dị thường Bouguer trên các điểm $p$ thuộc tập hợp theo công thức (11).

\section{Kết luận}

Trong nhiệm vụ xây dựng cơ sở dữ liệu dị thường trọng lực quốc gia trên đất liền với độ cao địa hình không vượt quá $1500 \mathrm{~m}$, một trong những bài toán phức tạp nhất là xác định mặt xu thế của các dị thường Bouguer trên các điểm trọng lực và sử dụng nó để nội suy xác định các giá trị dị thường Bouguer trên các đỉnh của các ô chuẩn thuộc cơ sở dữ liệu dị thường trọng lực quốc gia theo phương pháp kriging tổng quát. Trong bài báo khoa học này đã giải quyết hai vấn đề: Đánh giá độ chính xác của dị thường Bouguer từ mô hình xu thế và hoàn thiện thuật toán nội suy các giá trị dị thường Bouguer theo phương pháp kriging tổng quát dựa trên thuật toán bình sai truy hồi với phép biến đổi xoay $T^{-T}$ khi tính đến ưu điểm của thuật toán này khi giải quyết bài toán bình sai gián tiếp kèm các điều kiện và kiểm tra sự có mặt của các trị đo thô.

Các kết quả nghiên cứu trong bài báo khoa học này đã được sử dụng trong đề tài Khoa học và Công nghệ cấp Bộ Tài nguyên và Môi trường giai đoạn 2015 - 2018 với mã số TNMT.2016.07.02 "Nghiên cứu phương pháp xác định các giá trị dị thường trọng lực trên các đỉnh của các ô chuẩn trong cơ sở dữ liệu trọng lực quốc gia" ./.○

\section{Tài liệu tham khảo}

[1]. Duncan W.J. (1944). Some devices for the solution of large sets of simultaneous linear equations. The London, Edinburg and Dublin Philosophical Magazin and Journal of Science, Seventh Series, 35, pp. 660-670.

[2]. Goad, C. C., C. Ch. Tsherning, M. M. Chin, 1984. Gravity Empirical Covariance Values for the Continental United States. Journal of Geophysical Research, Vol. 89, No. B9, pp. 7962 - 7968.

[3]. Goovaerts P. (1997). Geostatistics for natural resources evaluation. New York, Oxford University Press, 483 p.

[4]. Marcin Ligas, Marek Kulczycki, 2014. Kriging approch for local height transformations. $J$. 
Geodesy And Cartography, Vol. 63, N01, pp. 25-37. Polish Academy of Sciences. Doi: 10.2478/geocart-2014-0002.

[5]. Hà Minh Hòa, 2015. Vấn đề giải hệ phương trình chuẩn với ma trận chuẩn không xác định dương trong bài toán xây dựng cơ sở dữ liệu dị thường trọng lực theo phương pháp kriging tổng quát. Tạp chí Khoa hoc Đo đạc và Bản đồ, số 25, tháng 09/2015, trg. 1-9.○

\section{Summary}

Construction of formula for accuracy estimation of trend surface and algorithm for interpolation of Bouguer anomalies by general kriging

\section{Ha Minh Hoa}

\section{Vietnam Institute of Geodesy and Cartography}

Usage of the Bouguer anomalies on gravimetric points for solving a techno - scientifisc task of interpolation for a determination of the Bouguer anomalies on cross - over points of cells in a grid of gravity anomaly database consists of a construction of a trend surface of those anomalies and interpolation by general kriging based on the constructed trend surface. This scientific article had solved two problems: Accuracy estimation of the Bouguer anomalies determined from the trend surface and perfection of algorithm of interpolation of the Bouguer anomalies on gravimetric points for the determination of the Bouguer anomalies on cross - over points of cells in a grid of gravity anomaly database by general kriging based on recurrent algorithm with rotation $T^{-T}$. Solution of the above mentioned two problems allowed to accomplish relatively complicated techno - scientific task in construction of the national gravity anomaly database. $\bigcirc$ 